\title{
Easiness of Use and Validity Testing of VS-SENSE Device for Detection of Abnormal Vaginal Flora and Bacterial Vaginosis
}

\author{
Gilbert G. G. Donders, ${ }^{1,2,3}$ Camila Marconi, ${ }^{4}$ and Gert Bellen ${ }^{1}$ \\ ${ }^{1}$ Femicare Clinical Research for Women, Lombardstraat 28, 3300 Tienen, Belgium \\ ${ }^{2}$ Department of Obstetrics and Gynaecology, Regional Hospital H Hart, Kliniekstraat 45, 3300 Tienen, Belgium \\ ${ }^{3}$ Department of Obstetrics and Gynaecology, University Hospital Gasthuisberg, Herestraat 49, 3000 Leuven, Belgium \\ ${ }^{4}$ Department of Pathology, Botucatu Medical School, São Paulo State University, Rubiao Jr. District, 18618-970 Botucatu, SP, Brazil \\ Correspondence should be addressed to Gilbert G. G. Donders, gilbert.donders@femicare.net
}

Received 29 March 2010; Revised 2 June 2010; Accepted 1 July 2010

Academic Editor: Ronald H. Gray

Copyright (๑) 2010 Gilbert G. G. Donders et al. This is an open access article distributed under the Creative Commons Attribution License, which permits unrestricted use, distribution, and reproduction in any medium, provided the original work is properly cited.

\begin{abstract}
Accessing vaginal $\mathrm{pH}$ is fundamental during gynaecological visit for the detection of abnormal vaginal flora (AVF), but use of $\mathrm{pH}$ strips may be time-consuming and difficult to interpret. The aim of this study was to evaluate the VS-SENSE test (Common Sense Ltd, Caesarea, Israel) as a tool for the diagnosis of AVF and its correlation with abnormal $\mathrm{pH}$ and bacterial vaginosis (BV). The study population consisted of 45 women with vaginal $\mathrm{pH} \geq 4.5$ and 45 women with normal $\mathrm{pH}$. Vaginal samples were evaluated by VS-SENSE test, microscopy and microbiologic cultures. Comparing with $\mathrm{pH}$ strips results, VS-SENSE test specificity was $97.8 \%$ and sensitivity of $91 \%$. All severe cases of BV and aerobic vaginitis (AV) were detected by the test. Only one case with normal pH had an unclear result. Concluding, VS-SENSE test is easy to perform, and it correlates with increased $\mathrm{pH}$, AVF, and the severe cases of BV and AV.
\end{abstract}

\section{Introduction}

Abnormal vaginal discharge is one of the most frequent complaints in women attending gynaecological clinics. Frequently abnormal discharge is related to abnormal vaginal flora (AVF), such as bacterial vaginosis (BV), trichomoniasis, and aerobic vaginitis (AV) [1-4]. Despite the unpleasant sensation reported by the women suffering of vaginal infections, these infections have been correlated with adverse pregnancy outcome [5-7], as well as with increased risk of sexually transmitted diseases such as HIV [8].

Increased $\mathrm{pH}$ is found in vaginal samples of most women with AVF. Determining vaginal $\mathrm{pH}$ is a useful screening tool for the diagnosis of AVF, but this procedure may be time consuming during gynaecological examination. Additionally, most practitioners measure vaginal $\mathrm{pH}$ using $\mathrm{pH}$ indicator strips, whose colour scale can be difficult to interpret [9]. Therefore the utilization of a fast and user-friendly test to detect abnormal $\mathrm{pH}$ indicative of AVF would be useful and could contribute for less doubtful results.
The VS-SENSE (Commom Sense Ltd, Caesarea, Israel) consists in a vaginal swab, which includes the nitrazine yellow $\mathrm{pH}$ indicator. It was developed aiming to detect abnormalities in the $\mathrm{pH}$ values and in the buffer capacity of the vaginal secretion, which can be indicative of vaginal infections, such as BV and trichomoniasis (see website http:/ /www.cs-commonsense.com/content.asp?pageid=93). In the presence of vaginal secretion with abnormal parameters, the swab colour changes resulting in a positive test. The initially yellow-coloured swab may turn into green or blue in the presence of abnormal $\mathrm{pH}(>4.7)$ and any slight change in the swab colour should be considered a positive result. According to the manufacturer, and it can be used by physicians during consultation or even by patients presenting abnormal vaginal discharge.

Therefore, the aim of this study was to evaluate the usefulness of the VS-SENSE test to detect an abnormal $\mathrm{pH}$ and as an additional tool for the physician or, by extension, even for the self-diagnosis by the patient herself of abnormal vaginal flora, as well as evaluate the correlation of this test with presence of bacterial vaginosis. 


\section{Material and Methods}

2.1. Study Population and Inclusion Criteria. From September until November 2009, 90 women attending the vulvovaginitis clinic at Gasthuisberg University Hospital, Leuven, Belgium, were included in the study. During the gynaecological examination, vaginal $\mathrm{pH}$ was routinely measured, microscopy of fresh vaginal fluid was performed, and vaginal cultures were taken routinely in all women attending the clinic. Subjects were included in the VS-SENSE accuracy study when the vaginal $\mathrm{pH}$ was equal or superior to 4.5 , while the next following patient whose $\mathrm{pH}$ was normal (between 3.6 and 4.4) was included as a control patient. Cut-off of $\mathrm{pH}=4.5$ was established based on one of the criteria for the BV diagnosis according to Amsel et al. [10]. If the next patient also had an abnormal $\mathrm{pH}$, the following 2 patients with normal $\mathrm{pH}$ were included as controls. Case and control groups were composed of 45 women each. Exclusion criteria were age below 18, pregnancy, menopausal status, menstruation, vaginal douching, treatment for vaginal infections in the previous 14 days, and sexual intercourse in the previous 12 hours.

2.2. Samples Collection. All samples were collected by a physician during gynaecological examination. With two fingers spreading the vulva, a polyethylene swab was gently inserted in the introitus and pushed up to the upper vault of the vagina. The swab was pressed on a $\mathrm{pH}$ strip (MachereyNagel GmbH \& Co, Duren, Germany) for 5 seconds and the material of the swab was spread on a glass for the microscopic evaluation. After this, the swab was placed on Amies transport medium for accessing the microbiologic culture profiles. The VS-SENSE test swabs were introduced in the vagina in a similar way allowing contact with vaginal content for 10 seconds. After removal from the vagina, the swabs were observed by 3 persons for determination of the final colour. According to the manufacturer's instructions, the originally light-yellow swab changes to green or blue after contact with samples with bacterial vaginosis, or it remains unchanged with yellow coloration in case of normal microflora. The colour of the swab was based on the concordant opinion of at least 2 observers. If all 3 observers agreed with the result, swab colour was defined as clear, if not, colour reading was defined as unclear. The first observer was aware if the sample belonged to case or control group while the 2 other observers were blinded. After analysing the swab colour, the first observer showed the sample to the other observers individually who communicate their opinion independently. Thus, the first observer compares the opinions and classifies them as clear or unclear.

\subsection{Microscopic Assessment of the Vaginal Microflora. Imme-} diately after collection of the smears, one droplet of saline was added to the glass and microscopic evaluation was performed in $\times 400$ magnification in a phase-contrast microscope (Leica MD1000, Wetzlar, Germany). The protocol for classification of AVF, aerobic vaginitis, full or partial bacterial vaginosis was done according toDonders [3]. Lactobacillary grades (LBG) were classified as LBG I when Lactobacillus morphotypes were predominant, LBG IIa as a normal lactobacillary pattern but with significant quantity of non-Lactobacillus morphotypes, LBG IIb when nonlactobacillary flora exceeded the quantity of Lactobacillus and finally the LBG III when the total replacement of the lactobacillary flora by other morphotypes was observed. Diagnosis of bacterial vaginosis was performed according to the extension of the typical anaerobe granular microflora in the sample. If the typical granular flora of BV-like bacteria was observed all over the smear or if there were more than $20 \%$ of epithelial cell covered with bacteria (clue-cells) it was diagnosed as "Full BV." When less than $20 \%$ of clue cells were observed or there were areas with streaks of BV-like bacteria mixed with other morphotypes, it was classified as "Partial BV" [6]. Aerobic vaginitis flora was defined as the presence of small bacilli and/or cocci in pairs or chains and scored according to the severity, taking into account the lactobacillary grade, pattern of the background, number of leucocytes, presence of toxic leucocytes, and presence of parabasal cells [4]. Candidiasis was diagnosed when unequivocal pseudohyphae and/or blastophores were detected on the smears and/or microbiology cultures resulted positive for Candida sp. Vaginal trichomoniasis was diagnosed if parasites with morphology and motility characteristic of Trichomonas vaginalis were visualized in the wet mount smears in the microscopic analysis. PCR for $T$. vaginalis was performed only when wet mount evaluation was inconclusive on its presence. $T$. vaginalis is infrequent in this population, its prevalence being $0.66 \%[11]$.

2.4. Microbiologic Cultures. The cotton swab from the vaginal wall was collected and transported to the microbiology laboratory in the Amies medium. Bacterial and yeast culture were performed in all samples and the microorganisms were semiquantified from $1+$ to $3+$, according to the number of yielded colonies.

2.5. Clinical Data. Oral consents were obtained from all patients and all relevant clinical data and medical history information were taken by filing a form specifically designed for the study. Information concerning the gynaecological history, days of last sexual intercourse, previous vaginal infections, and presence of current symptoms and discharge were collected. After informing about the need for IRB approval, we were told this was not necessary as the test was just a double check on a $\mathrm{pH}$ test that was done already routinely on every patient, comparable to testing a new device or colouring technique on samples in a chemical or pathology lab.

2.6. Statistical Analysis. $\mathrm{Chi}^{2}$ of Fisher exact were used as appropriate for categorical variables. Student's is used for normally distributed continuous variables like $\mathrm{pH}$ values and age, or Welch's equation if not normally distributed. 
TABle 1: Demographic and gynaecological characteristics of the women included in the study, divided into case and control groups, composed by 45 women each.

\begin{tabular}{|c|c|c|c|}
\hline Characteristics & Case $n(\%)$ & Control $n(\%)$ & $P$ value \\
\hline $\operatorname{Age}^{* a}$ & $37.2 \pm 11.9$ & $35.1 \pm 9.9$ & $P=.56$ \\
\hline \multicolumn{4}{|l|}{ Parity $^{b}$} \\
\hline Nulilparous & $26 / 43(60.8)$ & $30 / 44(68.2)$ & $P=.60$ \\
\hline Multiparous & $17 / 43(39.5)$ & $14 / 44(31.8)$ & $P=.60$ \\
\hline \multicolumn{4}{|l|}{ History $^{\mathrm{b}}$} \\
\hline Candida & $19(42.2)$ & $29(64.4)$ & $P=.057$ \\
\hline Recurrent candida & $10(22.2)$ & $19(42.2)$ & $P=.07$ \\
\hline BV & $11(24.2)$ & $1(2.2)$ & $P=.005$ \\
\hline AV & $17(37.8)$ & $2(4.4)$ & $P=.0003$ \\
\hline $\mathrm{pH}^{* \mathrm{a}}$ & $5.3 \pm 0.4$ & $3.9 \pm 0.3$ & $P<.0001$ \\
\hline \multicolumn{4}{|l|}{ Symptoms/complains ${ }^{\mathrm{b}}$} \\
\hline Burning & $13(28.9)$ & $12(26.7)$ & $P=1.0$ \\
\hline Itching & $10(22.2)$ & $10(22.2)$ & $P=.8$ \\
\hline Pain & $15(33.3)$ & $16(35.5)$ & $P=1.0$ \\
\hline Discharge & $21(46.7)$ & $10(22.2)$ & $P=.026$ \\
\hline
\end{tabular}

${ }^{*}$ Data expressed in mean \pm sd.

${ }^{a}$ Student's test (normal distribution) or Withney Rank Sum test (not normal distribution).

${ }^{\mathrm{b}} \mathrm{Chi}^{2}$ or Fisher exact test.

TABLE 2: Sensitivity and specificity of VS-SENSE test with pH less than 4.5 or more as discrimatie factors. Sensitivity: $82.2 \%$, Specificity: 97.8\%, Positive predictive value: 93.5\%, and Negative predictive value: 93.4\% (Bayes theorem).

\begin{tabular}{lccc}
\hline & Case group $(\mathrm{pH} \geq 4.5)$ & Control group $(\mathrm{pH}<4.5)$ & Total \\
\hline Positive & $37(82.2)$ & $1(2.2)$ & 38 \\
Negative & $8(17.8)$ & $44(97.8)$ & 52 \\
\hline Total & 45 & 45 & 90 \\
\hline
\end{tabular}

\section{Results}

In the period from September 2008 to March 2009, 227 women were seen at the vulvovaginitis clinic at Gasthuisberg University Hospital, Leuven, Belgium. Abnormal vaginal $\mathrm{pH}$ were found in $63(27.8 \%)$ of the patients. The evaluation with VS-SENSE test swabs were performed in vaginal samples from 45 women with $\mathrm{pH} \geq 4.5$ and from the subsequent 45 women with normal $\mathrm{pH}$ fitting the inclusion/exclusion criteria. Although additional 18 women presented abnormal $\mathrm{pH}$, their samples were not assessed by VS-SENSE test swabs by the presence of blood in the samples, recent intercourse, pregnancy or by their menopausal status (exclusion criteria).

Demographics and gynaecological data are presented in Table 1. Patients from the study group, and therefore presenting abnormal $\mathrm{pH}$ had significant higher rates of previous episodes of $\mathrm{BV}$ and $\mathrm{AV}$, and borderline lower rates of previous acute or chronic candidiasis.

As in the control group ( $\mathrm{pH}<4.5)$ only 1 false-positive case was detected, specificity of the test was as high as $97.8 \%$. In the case group $(\mathrm{pH} \geq 4.5)$, there were approximately $18 \%$ false negative cases accounting for a sensitivity of $82 \%$ (Table 2). There was divergence of opinion in between the 3 observers $18(20.0 \%)$ samples, thus considered unclear results. Unclear colour was observed only rarely in normal controls (1 case), while in the women with $\mathrm{pH}$ of 4.5 or above, green and blue swabs were considered unclear in approximately $1 / 3$ and $2 / 3$ of the cases, respectively (Table 3 ). Swabs presenting green colour had the highest percentage of unclear readings, with a disagreement among observers of over $40 \%$ of the cases (10/22 unclear green versus $4 / 52$ unclear yellow, $P=.0005)$, followed by blue readings $(4 / 16$ unclear blue versus $4 / 52$ unclear yellow, $P=.08$ ).

The comparison between VS-SENSE test and microscopy result of the vaginal smears is shown in Table 4 . From the total of 40 samples with normal flora, $3(7.5 \%)$ had positive swab results. In $15(30 \%)$ cases of AVF, the swab colour remained yellow indicating a false negative test. All 4 cases with severe AV were detected by VS-SENSE, but from the 23 samples presenting the diagnosis of mild AV, only 14 $(60.0 \%)$ had positive swab results. Similarly, all 11 cases with microscopic diagnosis of full BV were detected by the change of the swab colour, while only one of the 3 cases with partial BV was detected by the swab test. Twelve of $14(85.7 \%)$ women with candidiasis (by microscopy and/or microbiologic culture) had a negative result by the swab colour evaluation. All positive samples for candidiasis, but with negative result in the swab test had also normal LBG by microscopy (data not shown).

The microscopic evaluation allowed the identification of 2 cases of normal vaginal flora in the case group $(\mathrm{pH} \geq 4.5)$ which were also positive by VS-SENSE test (Table 5). Among 
TABLE 3: Results of VS-SENSE test and colour classification in clear and unclear, according to the agreement among observers.

\begin{tabular}{|c|c|c|c|}
\hline & Clear $n(\%)$ & Unclear $n(\%)$ & Total $(\%)$ \\
\hline Case group $(\mathrm{pH} \geq 4.5)$ & $28(62.2)$ & $17(37.8)^{* * *}$ & $45(100.0)$ \\
\hline Yellow & $5(11.1)$ & $3(6.7)$ & $8(17.8)$ \\
\hline Green & $12(26.7)$ & $10(22.2)$ & $22(48.9)$ \\
\hline Blue & $11(24.4)$ & $4(8.9)$ & $15(33.3)$ \\
\hline Control group $(\mathrm{pH}<4.5)$ & $44(97.8)$ & $1(2.2)$ & $45(100.0)$ \\
\hline Yellow & $43(95.6)$ & $1(2.2)$ & $44(97.8)$ \\
\hline Green & 0 & 0 & 0 \\
\hline Blue & $1(2.2)$ & 0 & $1(2.2)$ \\
\hline Total & $72(80.0)$ & $18(20.0)$ & $90(100.0)$ \\
\hline
\end{tabular}

${ }^{* * *} P<.0001$ versus control group. Fisher exact test.

TABLE 4: Results of VS-SENSE test showing the number of cases according to pattern of vaginal flora presented by microscopic evaluation, Candida infection and vaginal $\mathrm{pH}$ value.

\begin{tabular}{lccc}
\hline & Negative & Positive & Total \\
\hline Normal $^{\mathrm{a}}$ & $37(41.1)$ & $3(3.3)$ & $40(44.4)$ \\
Abnormal $^{\mathrm{b}}$ & $15(16.7)$ & $35(38.9)$ & $50(55.6)$ \\
\hline $\mathrm{AV}$ & & & \\
$\quad$ Mild & $9(10.0)$ & $14(15.6)$ & $4(25.6)$ \\
$\quad$ Moderate/severe & 0 & & $4(4.4)$ \\
\hline BV & $2(2.2)$ & $1(1.1)$ & $3(3.3)$ \\
$\quad$ Partial & 0 & $11(12.2)$ & $11(12.2)$ \\
$\quad$ Full & $4(4.4)$ & $2(2.2)$ & $6(6.6)$ \\
\hline AV + BV & $12(13.3)$ & $2(2.2)$ & $14(15.5)$ \\
Candida & & & \\
\hline pH & $44(48.9)$ & $37(41.1)$ & $45(50.0)$ \\
$\quad<4.5$ & $8(8.9)$ & $45(50.0)$ \\
\hline
\end{tabular}

${ }^{\mathrm{a}}$ Lactobacillary grade (LBG) I or IIa in microscopic evaluation; ${ }^{\mathrm{b}}$ LBG 0, IIb, or III in microscopic evaluation; ${ }^{\mathrm{C}}$ Presence of positive culture for Candida sp. or detection of sporae or hyphae by microscopy.

the samples with normal $\mathrm{pH}$ and negative VS-SENSE test, one case of LBG III was detected. In the control group, one case with $\mathrm{pH}$ of 3.8 and normal microscopy result revealed a blue final colour in the swab test. Blue colour was a stronger indicator of AVF (LBG III) than green, which by its turn reflects better abnormal results than yellow.

The microbiologic culture results showed overgrowth of bacteria in approximately 19\% of the samples (Table 6). Among the culture-positive cases, 4 presented normal results by microscopy, all of which were carrying grampositive Group-B streptococci (GBS) only. The remaining 13 had samples, had AVF on microscopic evaluation, and showed overgrowth of the gram-negative Escherichia coli and Klebsiella pneumoniae, in addition to GBS. Thirteen of 17 culture-positive samples also showed positive VS-SENSE test (76.5\%).

\section{Discussion}

Our findings revealed that VS-SENSE test is a highly specific test for detection of normal $\mathrm{pH}$ and vaginal flora, and can hence be considered an important tool for the reassurance of normal vaginal conditions. Furthermore, in normal women the yellow colour can easily be recognized, underlining its user-friendliness. Therefore, VS-SENSE test can be considered an easy to perform and very good test to exclude abnormal $\mathrm{pH}$ and consequently AVF.

The difficulty in the recognition of the swab colours was already reported by the group of Sobel et al., although their rate of doubtful cases was inferior to ours $[12,13]$. This may be due to the evaluation method adopted in the present study, in which the agreement of 3 observers was needed in order to call a test result unequivocally clear. Considering that green and blue colours had the highest rates of disagreement, differentiation between these 2 colours might not be relevant if both would equally represent abnormalities in $\mathrm{pH}$ and AVF. According to the manufacturer, any change in the swab colour should be considered as positive. Although numbers were small, our results did not seem to indicate that abnormal $\mathrm{pH}$, abnormal vaginal flora and bacterial vaginosis were more frequent in blue than in green test results. But as green colours were twice as frequent unclear than blue colours, it must be decided whether they are in such cases truly positive or not. Our data clearly indicate that most of the doubtful green and 
TABLE 5: VS-SENSE test and vaginal pH values according to the lactobacillary grade (LBG) determined by microscopic evaluation of fresh vaginal smears. Differences according to Kruscal-wallis test.

\begin{tabular}{|c|c|c|c|c|c|c|c|c|c|c|}
\hline \multirow[b]{3}{*}{ LBG } & \multicolumn{6}{|c|}{ Case $(n=45)$} & \multicolumn{4}{|c|}{ Control $(n=45)$} \\
\hline & \multicolumn{2}{|c|}{ Yellow } & \multicolumn{2}{|c|}{ Green } & \multicolumn{2}{|c|}{ Blue } & \multicolumn{2}{|c|}{ Yellow } & \multicolumn{2}{|c|}{ Blue } \\
\hline & $n(\%)$ & $\mathrm{pH}^{*}$ & $n(\%)$ & $\mathrm{pH}^{*}$ & $n(\%)$ & $\mathrm{pH}^{*}$ & $n(\%)$ & $\mathrm{pH}^{*}$ & $n(\%)$ & $\mathrm{pH}^{*}$ \\
\hline 0 & 0 & & $2(9.1)$ & $5,5 \pm 0,2$ & $1(6.7)$ & 5,6 & 0 & & 0 & \\
\hline I & 0 & & $1(4.6)$ & 4,8 & $1(6.7)$ & 5,3 & $35(79,5)$ & $3,9 \pm 0,3$ & $1(100.0)$ & 3,8 \\
\hline lIa & 0 & & 0 & & 0 & & $2(4,6)$ & 4,1 & 0 & \\
\hline Ilb & $4(50,0)$ & $5,0 \pm 0,3$ & $5(22,7)$ & $5,3 \pm 0,5$ & 0 & & $6(13,6)$ & $4,2 \pm 0,3$ & 0 & \\
\hline Ill & $4(50,0)$ & $4,8 \pm 0,3$ & $14(63,6)$ & $5,2 \pm 0,4$ & $13(86.6)$ & $5,6 \pm 0,4$ & $1(2,3)$ & 4,2 & 0 & \\
\hline Total & $8(17,8)$ & $5,0 \pm 0,4^{\mathrm{a}}$ & $22(48,9)$ & $5,2 \pm 0,4^{\mathrm{b}}$ & $15(33,3)$ & $5,6 \pm 0,4^{\mathrm{c}}$ & $44(97,8)$ & $3,9 \pm 0,3$ & $1(2,2)$ & 3,8 \\
\hline
\end{tabular}

$* \mathrm{pH}$ values expressed in mean $\pm \mathrm{sd}$.

a versus b: $P=.234$.

$\mathrm{b}$ versus c: $P=.005$.

a versus c: $P=.004$.

TABLE 6: Results of microbiologic cultures of vaginal samples from cases and controls with normal and abnormal microscopic evaluation, according to the VS-SENSE test result.

\begin{tabular}{|c|c|c|c|}
\hline Microscopy & $\begin{array}{c}\text { Positive culture }^{\mathrm{a}} \\
n(\%)\end{array}$ & $\begin{array}{c}\text { Negative culture } \\
n(\%)\end{array}$ & $\begin{array}{l}\text { Total } \\
n(\%)\end{array}$ \\
\hline Normal & $4(4,4)^{b}$ & $36(40,0)$ & $40(44,4)$ \\
\hline Yellow swab & $3(3,3)$ & $34(37,8)$ & $37(41,1)$ \\
\hline Green or blue swab & $1(1,1)$ & $2(2,2)$ & $3(3,3)$ \\
\hline Abnormal & $13(14,4)^{\mathrm{c}}$ & $37(41,2)$ & $50(55,6)$ \\
\hline Yellow swab & $1(1,1)$ & $14(15,6)$ & $15(16,7)$ \\
\hline Green or blue swab & $12(13,3)$ & $23(25,6)$ & $35(38,9)$ \\
\hline Total & $17(18,8)$ & $73(81,2)$ & $90(100)$ \\
\hline
\end{tabular}

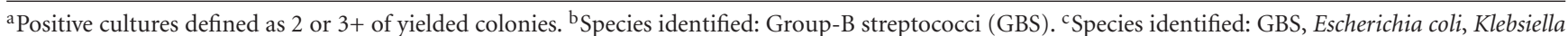
pneumoniae.

blue results were in fact found in women with abnormal flora and/or high $\mathrm{pH}$, indicating that in case of doubt, a slight discoloration from yellow to green or blue is to be considered positive. It is not known whether retesting shows less equivocal results.

Another issue is high rate of false-negative findings with the VS-SENSE test, according to our findings as high as around $30 \%$ of cases with AVF that would be missed if only performing the swab test. Since we included both LBG 0 and IIb in the definition of AFV in this paper, this wide definition could most likely contribute to a high false-negative rate. Detection and management of intermediately disturbed flora has caused much controversy in the literature $[3,14]$. Several studies show that intermediate flora relates to bad pregnancy outcome $[6,7,15,16]$. For this reason, the use of VS-SENSE to detect high-risk flora types during pregnancy should at the present time not be encouraged.

Concerning detection of more specific diseases, all severe cases of AV were properly detected by the VS-SENSE swab, while $60 \%$ of the mild cases were found. Also all cases of full $\mathrm{BV}$ were detected, but less so the cases with partial BV. So despite the VS-SENSE had a significant rate of false-negative cases for the detection of AVF, it was an efficient method to detect the most severe abnormalities of AV and BV. As could be expected, due to the absence of a link with abnormal flora or $\mathrm{pH}$, we provided confirmative evidence that VS-SENSE test is of no use in the detection of vaginal candidosis, nor colonisation with Candida. However, the statement of the manufacturer, that cases of vaginal candidosis should result in yellow swab colour, is not justified either, as this is only observed if also the LBG is normal. Due to the absence of cases with trichomoniasis in this series, the test value for this condition could not be tested.

Finally, given the $18 \%$ false-negative test results of the test system compared to increased $\mathrm{pH}$ value, however, one can discuss the necessity to repeat testing or additional tests in doubtful cases or when complaints are present. The data regarding the $\mathrm{pH}$ values and LBG found in each swab colour group shows that blue swabs correlate better with abnormal $\mathrm{pH}$ and AVF, when compared to green. The 2 cases with increased $\mathrm{pH}$ and VS-SENSE test positive, but classified as LBG I by microscopy, may partially be explained by recent sexual intercourse ( 24 hours), as found in one of the cases or by other influences on the $\mathrm{pH}$ without disturbing the bacterial flora. The VS-SENSE TEST is not advised for use in the event of recent sexual intercourse. The one sample with normal $\mathrm{pH}$ and negative swab test result in the swab test, but diagnosed as LBGIII and mild AV flora 
may be explained by the presence of coarse morphotypes of lactobacilli, such as the recently recognized Lactobacillus iners [17], that can be misinterpreted as astaphoid flora [18].

It was rather surprising that also aerobic microbiologic cultures results were associated with abnormal swab results. Not only $\mathrm{pH}$ or microscopy, known as likely indicators of an abnormal test, but also overgrowth of $E$. coli and $K$. pneumoniae were linked to a positive test. The clinical meaning of positive cultures on vaginal samples, however, is being a matter of discussion, since presence of positive culture is not a reason for treatment, unless in the presence of abnormal microscopy results and concordance with complaints.

We realize the positive predictive value of the test could be different in a random (screening) setting, as the lower prevalence of abnormal pH (e.g., 5\% to 10\%) underlines the importance of a high specificity. In this study, we choose for the scenario of cases versus controls, because it resembles better the real clinical setting. Indeed, we assume the test will mostly be used to test symptomatic women, not to screen asymptomatic women. Whereas specificity is more important in a setting with low prevalence (prevent unnecessary interventions), sensitivity should be high in a setting with high prevalence (not to miss to many cases).

It may need some explanation why it was chosen to use fresh wet mounts for AVF and BV testing and not the Gram stained Nugent's score. Wet mount reading allows better validation of lactobacillary flora grades than Gram stains, probably because of some lactobacilli get lost during the Gram fixation and coloring process, leading to a false increase in abnormal lactobacillary grades [19]. Furthermore, it allows better distinction between different flora types like BV, partial BV, and AV [4]. Finally, the study design using wet mounts correlates more to the everyday's practice-or at least what it should be-where sending of a specimen for Nugent is impractical due to many reasons [3].

Other points of care tests are available for the diagnosis of $\mathrm{BV}$ and abnormal pH. For example, the BVBlue test has been shown to be sensitive and specific [20], but their results are not directly comparable to ours. As discussed above, we envisioned not only to detect BV, but also other types of abnormal vaginal flora. For the VS Sense device tested in this study, a negative test excludes well the presence of disease, but some cases with AVF can still be missed.

In summary, we provide evidence that the VS-SENSE is an easy to perform test and it correlates well with increased $\mathrm{pH}$ and AVF, as well with severe cases of BV and $\mathrm{AV}$ infection. However, false negative results may occur and negative tests in the presence of clinical suspicion should prompt for retesting or additional testing or advice. Discerning between green and blue tests is not useful, but of both colours being more often unclear to read, the former has very often doubtful readings. According to our data, these doubtful cases should usually be seen as true positives.

\section{References}

[1] C. A. Spiegel, "Bacterial vaginosis," Clinical Microbiology Reviews, vol. 4, no. 4, pp. 485-502, 1991.

[2] J. Mashburn, "Etiology, diagnosis, and management of vaginitis," Journal of Midwifery and Women's Health, vol. 51, no. 6, pp. 423-430, 2006.

[3] G. G. G. Donders, "Definition and classification of abnormal vaginal flora," Best Practice and Research, vol. 21, no. 3, pp. 355-373, 2007.

[4] G. G. G. Donders, A. Vereecken, E. Bosmans, A. Dekeersmaecker, G. Salembier, and B. Spitz, "Definition of a type of abnormal vaginal flora that is distinct from bacterial vaginosis: aerobic vaginitis," BJOG, vol. 109, no. 1, pp. 34-43, 2002.

[5] H. Leitich, B. Bodner-Adler, M. Brunbauer, A. Kaider, C. Egarter, and P. Husslein, "Bacterial vaginosis as a risk factor for preterm delivery: a meta-analysis," American Journal of Obstetrics and Gynecology, vol. 189, no. 1, pp. 139-147, 2003.

[6] G. G. G. Donders, K. Van Calsteren, G. Bellen et al., "Predictive value for preterm birth of abnormal vaginal flora, bacterial vaginosis and aerobic vaginitis during the first trimester of pregnancy," BJOG, vol. 116, no. 10, pp. 1315-1324, 2009.

[7] G. G. G. Donders, B. Spitz, and A. Vereecken, "The ecology of the vaginal flora at first prenatal visit is associated with preterm delivery and low birth weight," The Open Infectious Diseases Journal, vol. 2, pp. 45-51, 2008.

[8] N. Sewankambo, R. H. Gray, M. J. Wawer et al., "HIV-1 infection associated with abnormal vaginal flora morphology and bacterial vaginosis," The Lancet, vol. 350, no. 9077, pp. 546-550, 1997.

[9] G. G. G. Donders, T. Caeyers, P. Tydhof, I. Riphagen, T. Van Den Bosch, and G. Bellen, "Comparison of two types of dipsticks to measure vaginal $\mathrm{pH}$ in clinical practice," European Journal of Obstetrics Gynecology and Reproductive Biology, vol. 134, no. 2, pp. 220-224, 2007.

[10] R. Amsel, P. A. Totten, C. A. Spiegel, K. C. S. Chen, D. A. Eschenbach, and K. K. Holmes, "Nonspecific vaginitis. Diagnostic criteria and microbial and epidemiologic associations," American Journal of Medicine, vol. 74, no. 1, pp. 14-22, 1983.

[11] C. Depuydt, I. Benoey, A. Vereecken, and G. G. G. Donders, "Prevalence and epidemiology of Trichomonas vaginalis detected by real time PCR in Flanders and its relation to HPV infection," Gynecologic and Obstetric Investigation. In press.

[12] A. Geva, J. Bornstein, M. Dan, H. K. Shoham, and J. D. Sobel, "The VI-SENSE-vaginal discharge self-test to facilitate management of vaginal symptoms," American Journal of Obstetrics and Gynecology, vol. 195, no. 5, pp. 1351-1356, 2006.

[13] J. D. Sobel, P. Nyirjesy, H. Kessary, and D. G. Ferris, "Use of the VS-sense swab in dagnosing vulvovaginitis," Journal of Women's Health, vol. 18, no. 9, pp. 1467-1470, 2009.

[14] G. G. G. Donders, "Bacterial vaginosis during pregnancy: screen and treat?" European Journal of Obstetrics Gynecology and Reproductive Biology, vol. 83, no. 1, pp. 1-4, 1999.

[15] G. G. G. Donders, A. Odds, A. Vereecken et al., "Abnormal vaginal flora in the first trimester, but not full-blown bacterial vaginosis, is associated with preterm birth," Prenatal and Neonatal Medicine, vol. 3, no. 6, pp. 588-593, 1998.

[16] P. E. Hay, R. F. Lamont, D. Taylor-Robinson, D. J. Morgan, C. Ison, and J. Pearson, "Abnormal bacterial colonisation of the genital tract and subsequent preterm delivery and late miscarriage," British Medical Journal, vol. 308, no. 6924, pp. 295-298, 1994. 
[17] E. De Backer, R. Verhelst, H. Verstraelen et al., "Quantitative determination by real-time PCR of four vaginal Lactobacillus species, Gardnerella vaginalis and Atopobium vaginae indicates an inverse relationship between L. gasseri and L. Iners," BMC Microbiology, vol. 7, article 115, 2007.

[18] R. Verhelst, H. Verstraelen, G. Claeys et al., "Comparison between Gram stain and culture for the characterization of vaginal microflora: definition of a distinct grade that resembles grade I microflora and revised categorization of grade I microflora," BMC Microbiology, vol. 5, article 61, 2005.

[19] G. G. G. Donders, A. Vereecken, A. Dekeersmaecker, B. Van Bulck, and B. Spitz, "Wet mount microscopy reflects functional vaginal lactobacillary flora better than gram stain," Journal of Clinical Pathology, vol. 53, no. 4, pp. 308-314, 2000.

[20] C. S. Bradshaw, A. N. Morton, S. M. Garland, L. B. Horvath, I. Kuzevska, and C. K. Fairley, "Evaluation of a point-of-care test, BVBlue, and clinical and laboratory criteria for diagnosis of bacterial vaginosis," Journal of Clinical Microbiology, vol. 43, no. 3, pp. 1304-1308, 2005. 


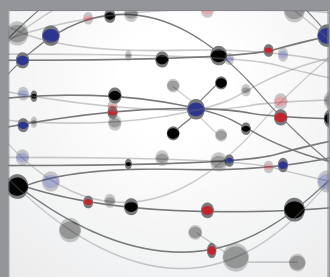

The Scientific World Journal
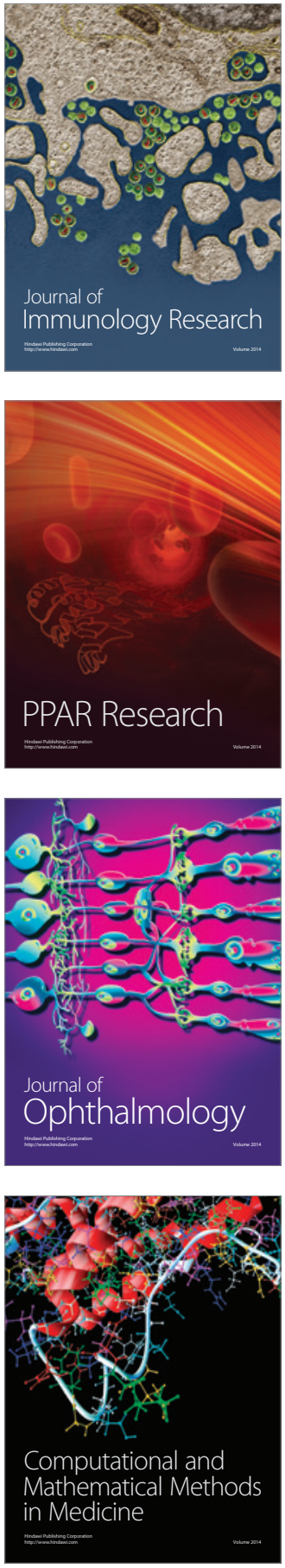

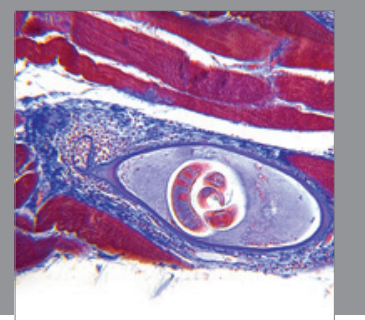

Gastroenterology

Research and Practice
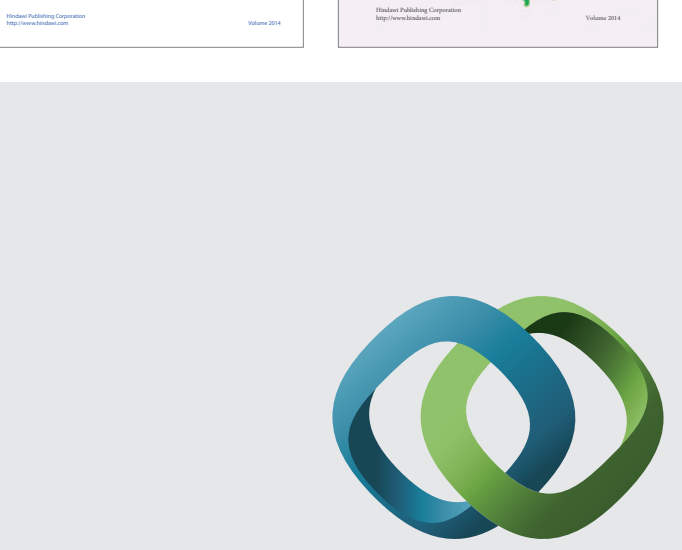

\section{Hindawi}

Submit your manuscripts at

http://www.hindawi.com
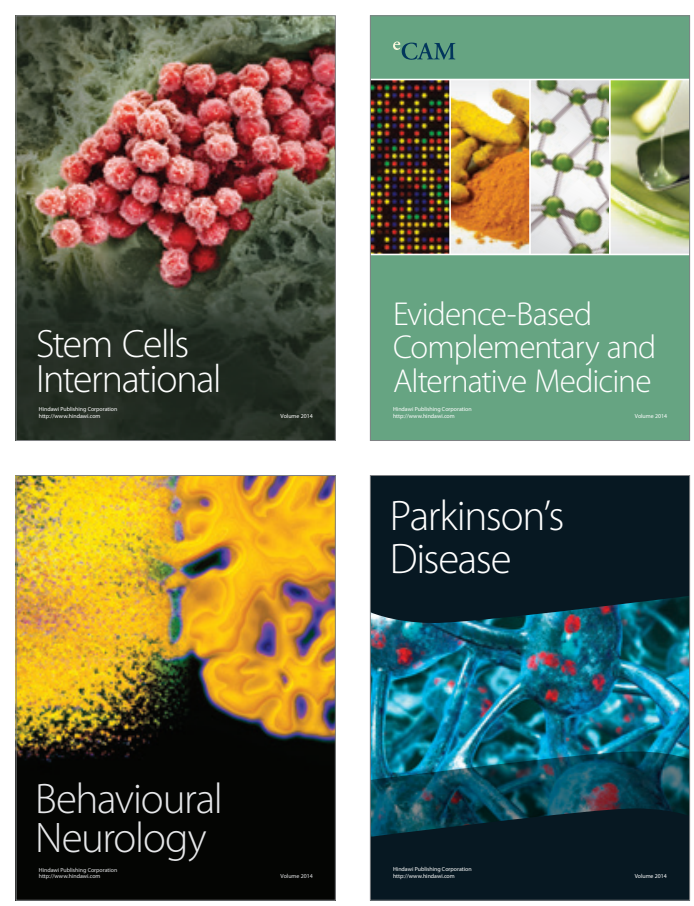

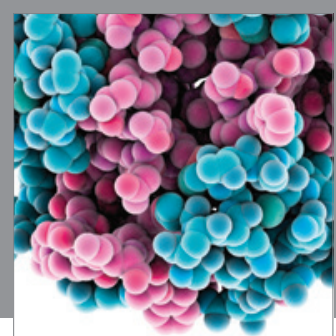

Journal of
Diabetes Research

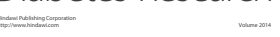

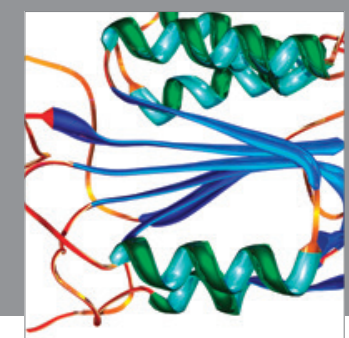

Disease Markers
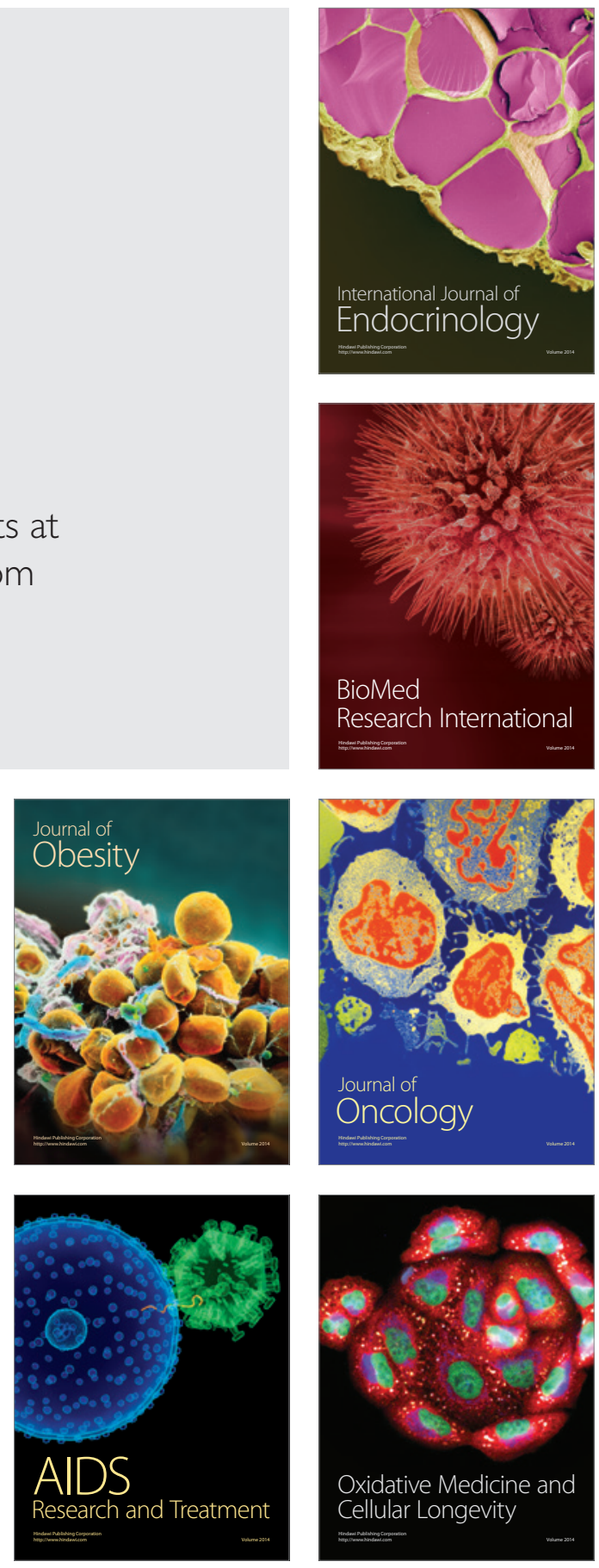